\title{
Besouros crisomelídeos em plantios de eucalipto na Zona da Mata Mineira
}

\author{
Chrysomelid beetles in eucalyptus plantations in Minas Gerais State, Brazil \\ Iris Cristiane Magistrali ${ }^{\mathrm{I}}$, Rodolfo Molinário de Souza ${ }^{\mathrm{II}}$, Norivaldo do Anjos ${ }^{\mathrm{III}}$, Cristiane Leal Duarte ${ }^{\mathrm{IV}}$
}

\begin{abstract}
Resumo
A cultura do eucalipto tem crescido nos últimos anos na Mesorregião da Zona da Mata Mineira. Entretanto, apesar dos fatores favoráveis para o estabelecimento de plantios de eucalipto nessa região, a ocorrência de besouros desfolhadores da família Chrysomelidae pode inviabilizar tais projetos florestais. Portanto, o objetivo deste trabalho foi registrar e caracterizar a ocorrência desses besouros em plantios de eucalipto localizados na Zona da Mata Mineira. Foi avaliado apenas um plantio por localidade com base no primeiro relato de ocorrência desses insetos. Em cada plantio, 100 árvores foram amostradas para obtenção do percentual de árvores atacadas. Verificou-se a presença de Costalimaita ferruginea, Agrianes validicornis e Metaxyonycha angusta injuriando árvores de eucalipto em Astolfo Dutra, Coimbra, Viçosa e Visconde do Rio Branco. Apenas Costalimaita ferruginea ocorreu em nível de surto nos quatro plantios vistoriados. Constatou-se que, em Visconde do Rio Branco, todas as árvores estavam atacadas por Costalimaita ferruginea e em Viçosa, Coimbra e Astolfo Dutra o percentual de ataque foi de 15\%, 46\% e 64\%, respectivamente. Os resultados obtidos neste trabalho servem de alerta para os produtores que já possuem plantios localizados na região da Zona da Mata Mineira e para aqueles que pretendem estabelecer novos plantios, assim como para o poder público que fornece assistência técnica aos pequenos produtores.
\end{abstract}

Palavras-chave: Besouros desfolhadores; Entomologia florestal; Eucalyptus; Produção

\begin{abstract}
Eucalyptus plantations have been growing in the last years in the Mesoregion of Zona da Mata Mineira, Minas Gerais state, Brazil. However, in spite of the favorable factors for the establishment of eucalypt plantations in this region, the occurrence of leaf-eating beetles of the family Chrysomelidae can make such forest projects unfeasible. Therefore, this work aimed to record and characterize the occurrence of leaf-eating beetles in eucalypt plantations located in Zona da Mata Mineira. One plantation by location was evaluated based in the first record of occurrence of the insects in the year 2008. In each plantation, 100 trees were sampled to obtain the frequency of damaged trees. The following beetles were observed: Costalimaita ferruginea; Agrianes validicornis and Metaxyonycha angusta, damaging eucalypt trees in Astolfo Dutra, Coimbra, Viçosa and Visconde do Rio Branco counties. Only outbreaks of Costalimaita ferruginea happened in the four plantations evaluated. In Visconde do Rio Branco, all the trees were damaged by Costalimaita ferruginea and in Viçosa, Coimbra and Astolfo Dutra 15\%, 46\% and 64\% were damaged, respectively. The results obtained in this work serve as an alert for authorities and forest growers acting in the Zona da Mata Mineira, Minas Gerais state.
\end{abstract}

Keywords: Leaf-eating beetles; Forest entomology; Eucalyptus; Production

\footnotetext{
${ }^{1}$ Engenheira Florestal, Doutora em Ciências Ambientais e Florestais, Universidade Federal Rural do Rio de Janeiro, Rod. 465, Km 7, CEP 23890-000, Seropédica (RJ), Brasil. irismagistrali@gmail.com (ORCID: 0000-0003-3563-3644)

II Engenheiro Florestal, Dr., Professor do Curso de Bacharelado em Engenharia Florestal, Campus Professora Cinobelina Elvas, Universidade Federal do Piauí, Av. Manoel Gracindo, km 1, Planalto Horizonte, CEP 64900-000, Bom Jesus (PI), Brasil. molinariodesouza@gmail.com (ORCID: 0000-0001-7346-8032)

III Engenheiro Florestal, PhD., Professor Titular do Departamento de Biologia Animal, Universidade Federal de Viçosa, Av. Purdue, s/n, CEP 36570-000, Viçosa (MG), Brasil. nanjos@ufv.br (ORCID: 0000-0002-4424-2955)

IV Engenheira Florestal, Especialista em Gestão Florestal, Servidora da Secretaria de Estado de Meio Ambiente e Desenvolvimento Sustentável do Estado de Minas Gerais, Rod. Ubá-Juiz de Fora, Km 2, Horto Florestal, CEP 36500-970, Ubá (MG), Brasil. eng.florestal@yahoo.com.br (ORCID: 0000-0001-8640-3107)
} 


\section{Introdução}

A cultura do eucalipto no Estado de Minas Gerais atualmente conta com cerca de 1.400 .00 ha plantados, ocupando o primeiro lugar em extensão de área no Brasil (ASSOCIAÇÃO BRASILEIRA DE PRODUTORES DE FLORESTAS PLANTADAS, 2011). Na Mesorregião da Zona da Mata Mineira, a expansão da eucaliptocultura tem sido em consequência das parcerias realizadas entre as prefeituras e os órgãos estaduais, como a Empresa de Assistência Técnica e Extensão Rural do Estado de Minas Gerais (EMATER), a Empresa de Pesquisa Agropecuária de Minas Gerais (EPAMIG), o Instituto Estadual de Florestas (IEF) e instituições federais de pesquisa que têm estimulado o uso do eucalipto como uma alternativa de renda e ocupação sustentável do solo, através de sistemas que integram lavoura, pecuária e floresta (EMPRESA DE PESQUISA AGROPECUÁRIA DE MINAS GERAIS, 2008). A expansão do Polo Moveleiro na região também tem estimulado o aumento de áreas com plantio de eucalipto, devido à demanda por essa matéria-prima por parte da indústria moveleira (VALE, 2004). Além disso, as condições climáticas, aliadas à topografia, fazem com que a exploração de madeira seja uma alternativa potencial para a região (OLIVEIRA, 1971; GOMES, 1986).

Apesar dos fatores favoráveis ao estabelecimento de plantios de eucalipto na Zona da Mata Mineira, é importante conhecer e monitorar os riscos bióticos que podem inviabilizar tais projetos florestais. Entre eles, destaca-se a ocorrência de besouros desfolhadores, principalmente daquelas espécies pertencentes à família Chrysomelidae, que são considerados um dos principais obstáculos para os produtores de eucalipto em todo o Brasil (ANJOS; MAJER, 2003). Os insetos adultos, pertencentes a essa família, são desfolhadores natos que, durante o comportamento alimentar, removem grande quantidade de área foliar, reduzindo o processo fotossintético, com consequências diretas no crescimento das árvores, o que pode ser convertido em perdas de ordem econômica. Portanto, devido ao crescente aumento na área de plantios de eucaliptos, aliado à carência de informações sobre besouros desfolhadores nessa região, se fazem necessários estudos que visem conhecer os insetos daninhos associados aos plantios de eucaliptos na Zona da Mata Mineira.

Os levantamentos de espécies de crisomelídeos realizados na Zona da Mata Mineira não são recentes. Schlottfeldt (1944a; 1944b) foi o primeiro a relatar a ocorrência de 36 espécies desta família, no município de Viçosa. Posteriormente, foram realizados trabalhos similares por Jolivet (1987), Anjos et al. (1995), Silveira (1996) e Gonçalves (2008). Porém, não há registro de espécies que são nocivas ao eucalipto, nessa mesorregião mineira, nem mesmo informações sobre suas distribuições geográficas. Assim, o objetivo deste trabalho foi notificar a ocorrência desses besouros em plantios comerciais de eucaliptos, nessa mesorregião de Minas Gerais, e caracterizar os danos causados às árvores.

\section{Material e Métodos}

O estudo foi desenvolvido a partir de vistorias realizadas em plantios de eucalipto de quatro municípios localizados na Mesorregião da Zona da Mata Mineira, no estado de Minas Gerais, que, segundo Golfari (1975), está situada no domínio da Floresta Atlântica e apresenta clima tropical de altitude (temperatura média de $18^{\circ} \mathrm{C}$, precipitação média anual de $1.500 \mathrm{~mm}$, com 2-4 meses secos por ano), relevo acidentado, com declividades que variam de 20 a $45 \%$ e altitudes que variam de 200 a 1.800

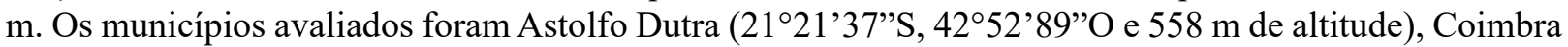

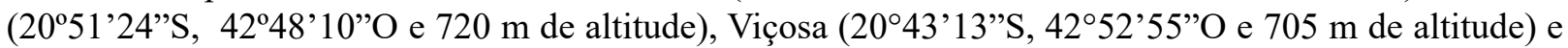
Visconde do Rio Branco ( $21^{\circ} 00^{\prime} 41^{\prime \prime} \mathrm{S}, 42^{\circ} 52^{\prime} 36^{\prime \prime} \mathrm{O}$ e $389 \mathrm{~m}$ de altitude). A descrição de cada plantio se encontra na Tabela 1.

As vistorias ocorreram no período de novembro a dezembro de 2008 e a escolha dos plantios foi realizada com base no primeiro relato espontâneo de ocorrência de besouros desfolhadores da família Chrysomelidae por proprietários rurais e por técnicos da EPAMIG. Nos locais vistoriados, adultos dessa família foram coletados para determinação da espécie através de comparações com exemplares depositados no Museu Regional de Entomologia da Universidade Federal de Viçosa (UFVB). 
Tabela 1 - Caracterização dos plantios de eucalipto atacados por besouros desfolhadores.

Table 1 - Characterization of eucalyptus plantations attacked by leaf-eating beetles.

\begin{tabular}{|c|c|c|c|c|}
\hline Localidade & Espécie plantada & Espaçamento (m) & $\begin{array}{c}\begin{array}{c}\text { Área } \\
\text { plantada }\end{array} \\
\text { (ha) }\end{array}$ & $\begin{array}{l}\text { Idade } \\
\text { (mês) }\end{array}$ \\
\hline Astolfo Dutra & Eucalyptus urophylla & $1,9 \times 2,5$ & 2,0 & 12,0 \\
\hline Coimbra & $\begin{array}{l}\text { Eucalyptus urophylla x. Eucalyptus } \\
\text { grandis }\end{array}$ & $6,0 \times 1,5$ & 11,0 & 8,0 \\
\hline Viçosa & Eucalyptus urophylla & $3,0 \times 2,0$ & 1,0 & 6,0 \\
\hline Visconde do Rio Branco & Eucalyptus urophylla & $2,5 \times 2,5$ & 23,7 & 12,0 \\
\hline
\end{tabular}

A confirmação dos danos causados pelos insetos às árvores de eucalipto foi realizada no campo através de observações diretas do comportamento daninho de cada espécie. Além disso, foi utilizado um sistema de amostragem para caracterizar os danos causados pelos crisomelídeos às árvores de eucalipto. $\mathrm{O}$ sistema consistiu em percorrer, em cada uma das quatro margens do plantio, 25 árvores na linha de plantio, no sentido da borda para o centro, conforme metodologia adaptada de Mendes (1999). A seleção da linha amostrada foi feita com base na "Técnica do Pior Foco", conforme preconizado por Anjos, Moreira e Della Lucia (1993). Em cada árvore mediu-se a intensidade de ataque, discriminada através de uma escala visual, adaptada de Mendes (2004), e definida por quatro notas ("0" = árvores sem sinal de ataque; "1" = desfolhamento menor que o terço superior da copa; " 2 " = desfolhamento do terço superior mais ponteiro principal atacado e " 3 " = desfolhamento maior que o terço superior da copa mais o ponteiro principal atacado).

As frequências relativas em cada classe visual de intensidade de ataque, para cada localidade, foram comparadas utilizando-se o teste de $\chi^{2}$. Para auxílio na interpretação dos resultados foi realizada uma análise de resíduo da tabela de contingência. Todas as análises foram obtidas através do programa Statistica v.7.0. (STATSOFT, 2004), adotando-se um nível de significância de 5\% de probabilidade.

\section{Resultados e Discussão}

A presença de besouros desfolhadores da família Chrysomelidae e as injúrias por eles causadas ao eucalipto foram constatadas nos quatro municípios da Zona da Mata vistoriados, e as espécies foram determinadas como sendo Costalimaita ferruginea (Fabricius, 1801) ("besouro-amarelo-do-eucalipto"), Agrianes validicornis (Chapuis, 1874) ("besouro-barriga-preta") e Metaxyonycha angusta (Perty, 1832) ("besouro-quatro-pintas"), todas pertencentes à subfamília Eumolpinae.

Em relação à distribuição geográfica e às plantas hospedeiras dessas espécies no estado de Minas Gerais, verificou-se que, para Costalimaita ferruginea, existia até o momento apenas um relato no estado realizado por Anjos (1992), que afirmou que esta espécie ocorre em 15 municípios. Entretanto, nenhum dos municípios citados por esse autor faz parte da Mesorregião da Zona da Mata e, além disso, os relatos não mencionam qualquer tipo de planta hospedeira para o besouro-amarelo. Desta maneira, é a primeira vez que se registra essa espécie associada a plantios de Eucalyptus urophylla S.T. Blake e híbrido de Eucalyptus urophylla x Eucalyptus grandis W. Hill ex Maiden no estado, sendo também o primeiro registro desta espécie na Zona da Mata Mineira. Verificou-se também a presença de ataque em goiabeiras próximas aos plantios de eucaliptos, no município de Astolfo Dutra, e em um goiabal localizado no município de Visconde do Rio Branco, sendo estes os primeiros registros desse inseto alimentando-se de Psydium guajava L. (Myrtaceae) em Minas Gerais.

A espécie Agrianes validicornis já havia sido relatada associada a clones de Eucalyptus urophylla, Eucalyptus camaldulensis Dehnh e Eucalyptus saligna Smith no município de Andrelândia (FERNANDES et al., 2005). Portanto, registra-se, pela primeira vez, a ocorrência dessa espécie em municípios da Zona da Mata Mineira. 
A espécie Metaxyonycha angusta foi registrada, primeiramente no estado, atacando eucaliptos no município de Três Pontas por Lima (1955) e, posteriormente, Fernandes (2004) constatou esse inseto associado a plantios de eucaliptos em Andrelândia, na Mesorregião Sul e Sudeste de Minas Gerais, e na Mesorregião do Campo das Vertentes nos municípios de Ibertioga e Santana do Garambéu. A partir de exemplares depositados em museus, o autor ampliou a área de ocorrência de Metaxyonycha angusta em Minas Gerais, incluindo os municípios de Santos Dumont, na Mesorregião Zona da Mata, e Patos de Minas, na Mesorregião do Triângulo Mineiro e Alto Paranaíba, mas não houve menção a espécies hospedeiras. Assim, este trabalho amplia a distribuição geográfica desse crisomelídeo no estado de Minas Gerais, sendo o primeiro registro desse inseto associado a Eucalyptus urophylla e híbrido de Eucalyptus urophylla vs. Eucalyptus grandis na Zona da Mata Mineira, nos municípios de Astolfo Dutra, Coimbra, Viçosa e Visconde do Rio Branco.

As três espécies ocorreram nos meses de novembro e dezembro, coincidindo com um período marcado por grande volume de chuvas e por altas temperaturas, na região de estudo. De fato, as condições climáticas parecem influenciar a dinâmica populacional de espécies da família Chrysomelidae. Gonçalves (2008), ao estudar a flutuação populacional de outros crisomelídeos presentes em uma área de regeneração natural, localizada no município de Viçosa, Minas Gerais, constatou que a temperatura e a precipitação pluviométrica afetaram positiva e significativamente a quantidade de espécies e de indivíduos adultos dessa família. A presença de Costalimaita ferruginea e de Metaxyonycha angusta em plantios de eucalipto sempre foi associada ao início das primeiras chuvas, conforme relataram Bondar (1953), Macedo (1975) e Fernandes (2004). Além disso, estudos realizados por Busoli (1979) e Souza et al. (2008) revelaram que os picos populacionais de adultos de Costalimaita ferruginea se correlacionaram positivamente com os picos de precipitação. Já Anjos (1992) definiu como crítico para a ocorrência desse crisomelídeo, na Região Sudeste do Brasil, o período que se estende de agosto de um ano a março do ano seguinte.

A presença de Costalimaita ferruginea foi a mais expressiva em todos os plantios avaliados, e se pôde verificar, inicialmente, árvores completamente infestadas e com sinais típicos do ataque por esse besouro, como folhas rendilhadas restando apenas a nervura principal, e em alguns casos árvores completamente desfolhadas. Também se observou ataque desse besouro no terço superior da copa das árvores. Já a presença de Agrianes validicornis foi mais intensa apenas em Astolfo Dutra, nos demais municípios sua ocorrência foi esporádica e menos intensa, assim como foram menores a ocorrência e as injúrias causadas por Metaxyonycha angusta.

No plantio localizado no município de Astolfo Dutra constatou-se que o foco maior do ataque de Agrianes validicornis encontrava-se na parte mais baixa do terreno, em reboleira, tendo sido observadas 37 árvores atacadas por este besouro, sendo que, em apenas uma árvore, foram coletados mais de 100 besouros em plena atividade daninha sobre os eucaliptos. Na parte superior do plantio e em uma de suas laterais, constataram-se besouros de Agrianes validicornis e de Costalimaita ferruginea utilizando-se da mesma árvore para alimentação, esconderijo e acasalamento.

Os adultos de todas as espécies de crisomelídeos encontradas alimentavam-se de árvores com até um ano de idade, danificando as extremidades dos ramos como ponteiros tenros, folhas novas e folhas do terço superior das árvores. Percebeu-se que folhas menos tenras também foram atacadas, sendo observado que os desfolhamentos nas árvores ocorreram predominantemente de cima para baixo, corroborando as observações feitas por Anjos (1992) e Fernandes (2004). Contatou-se que os adultos de Costalimaita ferruginea e Agrianes validicornis podem consumir todo o limbo foliar, restando apenas a nervura principal. Este é o primeiro registro de Agrianes validicornis em nível de surto no Brasil. Essa espécie mostrou-se extremamente daninha às árvores de eucalipto no campo. Por se tratar de uma espécie que já havia sido relatada na Mesorregião Sul e Sudoeste de Minas Gerais (FERNANDES, 2004), diferentemente da relatada no presente trabalho, infere-se que esse inseto em longo prazo pode vir a ocorrer nas demais regiões do estado em forma de surtos populacionais, danificando plantios de eucalipto.

Como Costalimaita ferruginea foi a única espécie que ocorreu concomitantemente em nível de surto nos quatro plantios vistoriados, os resultados a seguir são referentes à quantificação dos danos causados por esse besouro. A partir das avaliações realizadas, constatou-se que em Visconde do Rio Branco todas as árvores estavam atacadas, e em Viçosa, Coimbra e Astolfo Dutra o percentual de árvores atacadas foi de $15 \%, 46 \%$ e 64\%, respectivamente, conforme (Figura 1). Em Visconde do Rio Branco, observou-se que

Ci. Fl., Santa Maria, v. 29, n. 1, p. 425-431, jan./mar., 2019 
algumas árvores no plantio estavam com sintomas de deficit hídrico, além disso havia nas proximidades um pequeno plantio de goiabeiras, que também são um hospedeiro nativo para o besouro-amarelo. Esses dois fatores podem ter contribuído para a maior incidência de árvores atacadas em relação aos demais municípios, em que tal situação não foi observada.

\section{Figura 1 - Variação na intensidade de ataque causadas por Costalimaita ferruginea em plantio de eucaliptos no estado de Minas Gerais. Nov/Dez 2008.}

Figure 1 - Variation in the intensity of damage caused by Costalimaita ferruginea in eucalyptus plantations, Minas Gerais state, Brazil. Nov/Dec 2008.

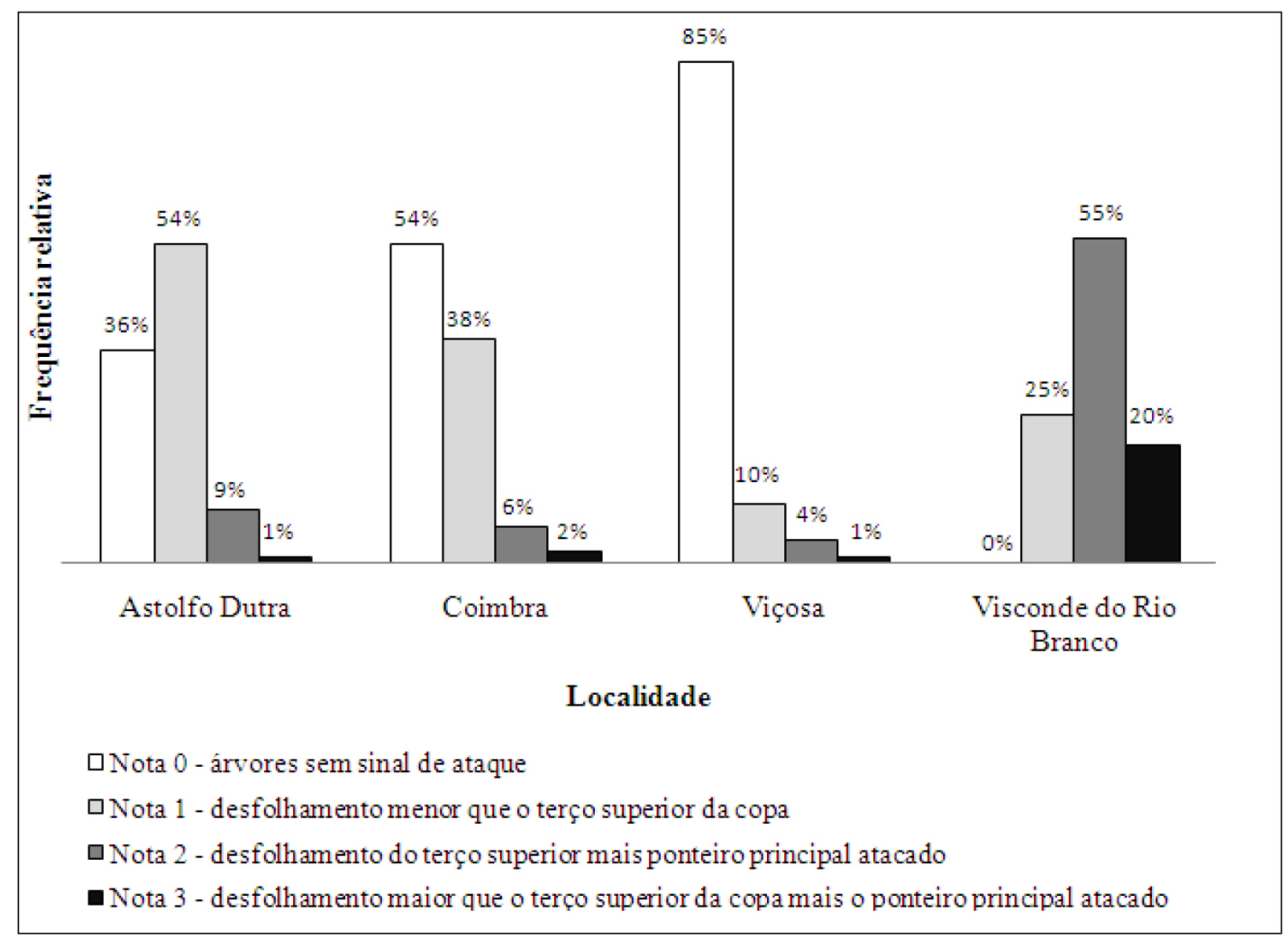

As distribuições percentuais por nota de intensidade de ataque diferiram significativamente entre as localidades $\left(\chi^{2}=259,9 ;\right.$ g.l $\left.=15 ; \mathrm{p}<0,00001\right)$, sugerindo uma relação entre esta variável e o local do surto. Pela análise de resíduo, as frequências relativas observadas em Visconde do Rio Branco para as notas 0,2 e 3 foram as que, significativamente, mais contribuíram para o valor do $\chi^{2}$ (resíduo = $-10,08 ; 10,85$ e 11,79 , respectivamente), enquanto para a nota 1 , a frequência observada para Viçosa foi significativamente maior (resíduo $=5,52$ ). Apenas na nota 0 , em Astolfo Dutra, os percentuais observados não foram significativamente diferentes daqueles esperados ao acaso (resíduo $=-1,80$ ).

Mendes (2004), ao estudar os efeitos do ataque de Costalimaita ferruginea em árvores de Eucalyptus grandis, com idade de sete a nove meses, verificou, em relação aos níveis de intensidade de ataque (adotadas aqui como nota 1, 2 e 3), perdas na produção de madeira iguais a $11,0 \%, 14,4 \%$ e 43,6\%, respectivamente. Em relação a essa variável, o autor definiu como nível de dano econômico um percentual de $6 \%, 3 \%$ e $1 \%$ para as notas 1,2 e 3 , respectivamente. Considerando essas informações, pode-se observar que para todas as três notas, o percentual encontrado neste trabalho, para os quatro municípios, foi superior ao nível de dano econômico estipulado por aquele autor.

Uma vez que Costalimaita ferruginea vem se destacando, no Brasil, como a principal espécie de besouro capaz de causar prejuízos expressivos aos plantios de eucalipto (ANJOS, 1992; MENDES; ANJOS; CAMARGO, 1998; MENDES, 2004, SOUZA et al., 2008), devido principalmente à frequência e periodicidade dos surtos, os resultados obtidos neste trabalho reforçam essa afirmativa e servem de alerta 
para os atuais e futuros produtores de eucalipto na região da Zona da Mata Mineira, além de servir de atenção para o setor público que tem estimulado o uso do eucalipto nessa região. Também serve de alerta a presença de Agrianes validicornis e Metaxyonycha angusta. A primeira por ter sido observada em nível de surto no município de Astolfo Dutra e a segunda, apesar de não ter sido registrada em nível de surto, já foi registrada por Fernandes (2004) causando grandes prejuízos em plantios de outras regiões do estado de Minas Gerais, que não a Zona da Mata.

\section{Conclusão}

Registra-se, para a Zona da Mata Mineira, a ocorrência de importantes besouros-desfolhadores da família Chrysomelidae que podem efetivamente comprometer os plantios com essa essência florestal na região. Além disso, os surtos de Costalimaita ferruginea foram caracterizados, revelando um alto percentual de árvores atacadas.

\section{Agradecimentos}

Aos colegas do Laboratório de Pragas Florestais da Universidade Federal de Viçosa pelas sugestões no presente trabalho. Ao biólogo Dinarte Gonçalves pela confirmação das espécies. À CAPES pelo apoio financeiro.

\section{Referências}

ASSOCIAÇÃO BRASILEIRA DE PRODUTORES DE FLORESTAS PLANTADAS. Anuário Estatístico da ABRAF 2011: ano base 2010. Brasília: ABRAF, 2011. 140 p. Disponível em: http://www.abraflor.org.br/ estatisticas/ABRAF11/ABRAF11-BR.pdf. Acesso em: 12 jan. 2012.

ANJOS, N. Taxonomia, ciclo de vida e dinâmica populacional de Costalimaita ferruginea (Fabr., 1801) (Coleoptera: Chrysomelidae), praga de Eucalyptus spp. (Myrtaceae). 1992. 165 f. Tese (Doutorado em Entomologia) - Escola Superior de Agricultura "Luiz de Queiroz", Piracicaba, 1992.

ANJOS, N. et al. Chrysomelidae de Viçosa. In: CONGRESSO BRASILEIRO DE ENTOMOLOGIA, 15., 1995, Caxambu. Anais... Caxambu: SBE, 1995. p. 777.

ANJOS, N.; MAJER, J. D. Leaf-eating Beetles in Brazilian eucalypt plantations. Curtin: School of Environmental Biology, 2003. 33 p. (Bulletin, 23).

ANJOS, N.; MOREIRA, D. D. O.; DELLA LUCIA, T. M. C. Manejo integrado de formigas cortadeiras em reflorestamentos. In: DELLA LUCIA, T. M. C. (Ed.). As formigas cortadeiras. Viçosa, MG: Folha de Viçosa, 1993. p. 212-241.

BONDAR, G. Pragas novas nas plantas do Brasil. Boletim do Campo, Rio de Janeiro, v. 9, n. 61, p. 20-24, 1953.

BUSOLI, A. C. Levantamento, constância e flutuação populacional de alguns insetos coletados com armadilha luminosa em Jaboticabal. 1979. 100 f. Dissertação (Mestrado em Entomologia) - Escola Superior de Agricultura “Luiz de Queiroz”, Piracicaba, São Paulo, 1979.

EMPRESA DE PESQUISA AGROPECUÁRIA DE MINAS GERAIS. Eucalipto - Base para a sustentabilidade da siderurgia. [2008]. Disponível em: http://www.epamig.br/index.php?option=com_cont ent\&task=view\&id=532\&Itemid=68. Acesso em: 16 set. 2010.

FERNANDES, L. C. Biologia de Metaxyonycha angusta (Perty) (Coleoptera: Chrysomelidae) e efeitos do seu ataque em eucaliptos, num sistema agroflorestal. 2004. 86 f. Dissertação (Mestrado em Entomologia) Universidade Federal de Viçosa, Viçosa, MG, 2004.

FERNANDES, L. C. et al. Ocorrência de Agrianes validicornis (Chapuis) (Coleoptera: Chrysomelidae) em cultura de eucaliptos consorciada. In: SIMPÓSIO DE ATUALIDADES EM PROTEÇÃO FLORESTAL, 2., 2005, Blumenau. Anais... Blumenau: Universidade Regional de Blumenau, 2005. 
GOLFARI, L. Zoneamento ecológico do Estado de Minas Gerias para reflorestamento. Belo Horizonte: CPFRC, 1975. 65 p. (Série Técnica, 3).

GOMES, S. T. Condicionantes da modernização do pequeno agricultor. 1986. 210 f. Tese (Doutorado em Economia) - Faculdade de Economia e Administração, Universidade de São Paulo, São Paulo, 1986.

GONÇALVES, D. Levantamento das espécies einfluência de variáveis climáticas sobre populações de Chrysomelidae. 2008. 99 f. Dissertação (Mestrado em Entomologia) - Universidade Federal de Viçosa, Viçosa, MG, 2008.

JOLIVET, P. E. Remarques sur la biocenose des Coelomera Chevrolat avec la description d'une nouvelle espece du Bresil (Coleoptera: Chrysomelidae: Galerucinae). Bulletin Mensal de lia Sociéte Linnéene de Lyon, Paris, v. 56, n. 8, p. 255-279, 1987.

LIMA, A. M. C. Insetos do Brasil. [S.1.]: Escola Nacional de Agronomia, 1955. t. 9. 288 p.

MACEDO, P. Estudo das principais pragas das ordens Lepidoptera e Coleoptera dos eucaliptais do Estado de São Paulo. 1975. 87 f. Dissertação (Mestrado em Entomologia) - Escola Superior de Agricultura "Luiz de Queiroz", Piracicaba, 1975.

MENDES, J. E. P.; ANJOS, N.; CAMARGO, F. R. A. Monitoramento do besouro-amarelo. Folha Florestal, Viçosa, MG, v. 91, p. 8-9, 1998.

MENDES, J. E. P. Efeitos do ataque de Costalimaita ferruginea (Fabr.) (Coleoptera: Chrysomelidae) sobre o crescimento e produção de Eucalyptus grandis Hill ex Maiden. 2004. 49 f. Tese (Doutorado em Entomologia) - Universidade Federal de Viçosa, Viçosa, MG, 2004.

MENDES, J. E. P. Nível de dano e impacto do desfolhamento por Costalimaita ferruginea (Fabr.) (Coleoptera: Chrysomelidae) em Eucalyptus grandis Hill ex Maiden. 1999. 99 f. Dissertação (Mestrado em Entomologia) - Universidade Federal de Viçosa, Viçosa, MG, 1999.

OLIVEIRA, A. J. Análise econômica da exploração florestal e sua combinação com outras atividades, pela programação linear, Zona da Mata, Minas Gerais. 1971. 171 f. Dissertação (Mestrado em Economia Rural) - Universidade Federal de Viçosa, Viçosa, MG, 1971.

SCHLOTTFELDT, C. S. Insetos encontrados em plantas cultivadas e comuns - Viçosa, Minas Gerais. Revista Ceres, Viçosa, MG, v. 6, n. 31, p. 52-65, 1944a.

SCHLOTTFELDT, C. S. Insetos encontrados em plantas cultivadas e comuns - Viçosa, Minas Gerais. Revista Ceres, Viçosa, MG, v. 6, n. 32, p. 108-126, 1944 b.

SILVEIRA, R. D. Biologia de Coelomera lanio (Dalman) (Coleoptera: Chrysomelidae: Galerucinae). 1996. 80 f. Dissertação (Mestrado em Entomologia) - Universidade Federal de Viçosa, Viçosa, MG, 1996.

SOUZA, R. M. et al. Condições climáticas associadas com a ocorrência de Costalimaita ferruginea (Fabricius) em plantios de eucaliptos. In: SIMPÓSIO DE MEIO AMBIENTE, 5., 2008, Viçosa, MG. Anais... Viçosa, MG: Centro Brasileiro para Conservação da Natureza e Desenvolvimento Sustentável, 2008.

STATSOFT. Statistica (data analysis software system). Version 7, 2004. Disponível em: www.statsoft.com.

VALE, R. S. Agrossilvicultura com eucalipto como alternativa para o desenvolvimento sustentável da Zona da Mata de Minas Gerais. 2004. 115 f. Tese (Doutorado em Ciência Florestal) - Universidade Federal de Viçosa, Viçosa, MG, 2004. 